\title{
Guidance and Navigation Challenges for a Mars Ascent Vehicle
}

\author{
Evan Anzalone \\ NASA/MSFC \\ MSFC/EV42 \\ Huntsville, AL 35812 \\ evan.j.anzalone@nasa.gov
}

\author{
Dane Erickson \\ NASA/MSFC \\ MSFC/EV42 \\ Huntsville, AL 35812 \\ dane.w.erickson@nasa.gov
}

\author{
Jason Everett \\ NASA/MSFC \\ MSFC/EV42 \\ Huntsville, AL 35812 \\ jason.m.everett@nasa.gov
}

\author{
Joey Powers \\ NASA/MSFC \\ MSFC/EV42 \\ Huntsville, AL 35812 \\ joey.powers@nasa.gov
}

\begin{abstract}
This work presents studies and analysis in support of a Mars Ascent Vehicle as part of a Martian Sample Return campaign. The vehicle design has been ongoing, with rapid development of a 6 Degree of Freedom simulation to capture full vehicle dispersions and integrated performance of vehicle, guidance, navigation and control. The maturation of this simulation is presented to provide an overview of its capabilities added over the past year of effort. The results describe in detail guidance algorithm development to increase the system's robustness to thrust sensitivities. Navigation performance and sensitivity analysis are included to describe the capabilities of the current design as well as identify primary drivers of insertion performance. Lastly, integrated vehicle 6DOF statistical results are presented to provide insight into the nominal performance of the current vehicle and insight into system-level drivers. Future work is described to outline the continuing maturation and development of the MSR MAV ascent vehicle.
\end{abstract}

\section{TABLE OF CONTENTS}

1. INTRODUCTION 1

2. SIMULATION EVOLUTION ........................................ 1

3. GUIDANCE DESIGN ..................................................... 2

4. NAVIGATION DESIGN ............................................ 4

5. INTEGRATEd VEHICLE PERFORMANCE.................. 9

6. CONCLUSIONS........................................................... 13

7. FUTURE WORK ................................................... 13

ACKNOWLEDGEMENTS .......................................... 13

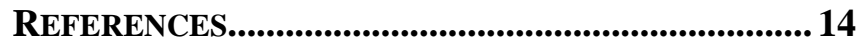

BIOGRAPHY .......................................................... 14

\section{INTRODUCTION}

As part of the next phase of Martian planetary science, the Mars 2020 rover includes the capability to collect samples of local surface material and prepare them for collection and return to Earth as part of a Martian Sample Return architecture. This concept of operations includes a lander housing a rover to collect the samples, an ascent vehicle to launch them into Martian orbit, and a separate orbiter which will rendezvous, capture, and return the samples to Earth. The focus of this work is in regards to the specific Guidance and Navigation challenges of launching a rocket from another's planet surface. The need for landing an integrated system consisting of these elements pushes the bounds of current capability. The long duration of the mission restricts the

\section{U.S. Government work not protected by U.S. copyright}

potential propellant options, focusing on solid or storable, liquid or hybrid systems. Uncertainties in propellant performance of the solid system bring forth a need for closed loop guidance algorithms that can manage excess energy. This research discusses the implementation and assessment of flight algorithms to meet the insertion requirements for the vehicles under consideration. Particular focus is placed on algorithms with energy management capability as well as full-closed solutions using traditional launch vehicle algorithms, such as Powered Explicit Guidance. The performance capability of these algorithms is assessed against the two reference solid and hybrid propulsion architectures against algorithm complexity, robustness, and insertion performance while simulating vehicle disturbances within a six degree of freedom simulation environment.

With the need for constrained mass and closed-loop guidance, the navigation system also faces conflicting requirements of performance vs. size. For closed-loop guidance approaches, any navigation errors directly correlate into insertion error. As such, a high quality solution is required. Due to the volume and mass limitations of the integrated Martian lander's capability, navigation-grade solutions are not feasible. Additionally, the system needs to be robust to the launch environment and highly reliable. In order to meet these requirements, lower grade inertial measurement units (IMU) must be used for inertial navigation. This study provides a review of the potential IMU options available and their performance with an integrated vehicle for the ascent trajectories. Sensitivity studies show the need for an accurate initial attitude prior to launch. In order to attain this, gyrocompassing or external measurements must be used. The capability of the potential sensors is assessed in detail. Lastly, in order to reduce mass and maintain insertion accuracy, this paper also considers the use of small size star trackers to perform attitude updates, providing an integration approach and description of the tradeoff between IMU accuracy/weight with enhanced robustness from a star tracker. The additional constraints in this use case are also described. Combined with guidance algorithm development, this paper provides an overview of the challenges and potential solutions to successful rocket flight from the Martian surface

\section{Simulation EvOLUTION}

Successful retrieval of Martian surface samples from orbit around Mars depends heavily on the orbital insertion 
accuracy of the ascent vehicle. Such an ascent vehicle must be capable of delivering the payload to the target orbit (within retrieval vehicle tolerances) under a variety of unpredictable conditions. Though not limited to this list, these dispersions can be variations in launch azimuth or elevation, atmospheric dust or wind conditions, solid rocket motor total impulse delivered, IMU orientation error, thrust misalignments, etc. The determination of whether a notional vehicle design can meet such performance demands requires both a 6 degree-offreedom (6DOF) simulation capable of including these varying conditions, and a GNC architecture capable of adapting to the conditions. Previous studies employed the original Mars Ascent vehicle aNalysis Tool In Simscape (MANTIS) [1] tool to support detailed vehicle requirements development and analysis. As vehicle concepts matured however, additions and upgrades to MANTIS were required in order to better understand vehicle performance across the spectrum of dispersed parameters, such as engine dispersions and launch day uncertainties.

As discussed in [1], MANTIS is a 6DOF ascent propagation tool created using Simscape Multibody [2]. The high level architecture of MANTIS is a plant block which propagates and integrates the dynamics, a sensor block to interpret signals from the plant and feed to GNC (guidance, navigation, and control), and the GNC block which uses measurements from the sensors to determine which signals to send forward to the plant: thrust vector commands, RCS (reaction control system) commands, staging commands (if applicable), and engine startup/shutdown commands (if applicable).

Several upgrades to the plant model were required to better assess 6DOF vehicle performance in dispersed conditions. The standard atmosphere model was replaced with an interface to incorporate the atmosphere and winds outputs from an external call to Mars-GRAM 2010 (Mars Global Reference Atmospheric Model 2010) [3]. Propulsion and mass propagation were upgraded to incorporate guidancecommanded staging and variable stage-two ignition timing for the solid motor vehicle, and variable startup/shutdown times and variable motor transients for the hybrid motor vehicle. Various changes throughout the plant also enabled the introduction of off-nominal conditions in initial mass, moments of inertia, CG location, winds, atmosphere, motor performance, and aerodynamics, all controlled via userdefined input flags.

The majority of the upgrades however, were in the sensor and GNC blocks for both vehicles. The original sensor block was replaced with a more detailed IMU model, and the capability to switch between IMU models via user input flags. A star tracker model was also added. These updates facilitate quicker turn-around in performing navigation sensitivity analysis. The guidance block was overhauled from the original MANTIS framework to allow for the inclusion of updated algorithms. These updates were implemented in early to mid-2019 in advance of a vehicle design cycle and analysis cycle.. Guidance and control are now managed according to flight phase, and guidance undergoes the following process:

1. Receive state information from navigation

2. Calculate current orbital elements

3. Update the phase manager

4. Load appropriate guidance targets based on phase

5. Update the time-to-ignition calculation (if applicable) for the second burn

6. Perform closed loop guidance calculations (if applicable)

7. Transform the guidance command to a controller command

8. Send the appropriate commands forward

In the case of the solid motor vehicle, guidance also determines when to drop the first stage inert mass and ignite the second stage. In the case of the hybrid motor vehicle, guidance determines the engine startup/shutdown commands to send to the plant. Various options for stage one and stage two guidance are also easily changed via input flags, enabling rapid assessment of the effects of different combinations of implemented guidance algorithms. Guidance targets are derived from optimal 3DOF trajectories using NASA Langley Research Center's POST2 (Program to Optimize Simulated Trajectories) [4].

In addition to upgrades to the base simulation, additional functionality was added to enable rapid turn-around of linear sensitivity studies and statistically-based dispersion studies. This additional capability allowed analysis of the orbital insertion effects of dispersing a range of parameters throughout the plant, sensor, and GNC models to simulate off-nominal conditions and assess orbital dispersion results through Monte Carlo analysis.

These upgrades to the MANTIS simulation enable a more detailed, dispersible simulation of the vehicle mode. In this way variable guidance algorithms, subsystem mass properties, motor properties, IMU models, and control laws can be tested and assessed in a dispersed environment.

\section{GUIDANCE DESIGN}

The importance of accurate orbital insertion mandates effective guidance strategies capable of responding to unexpected dispersions in vehicle and/or environmental parameters outside of pre-flight assumptions. In addition, responding to unpredicted conditions requires performance margin. For example, higher than anticipated drag and offnominal launch azimuth require additional delta-v capability in the vehicle in order to fly the sub-optimal trajectory required to insert into the target orbit. This performance margin manifests itself in different ways for each vehicle. The solid motor vehicle must burn all propellant available once a specific motor is ignited, and thus will expend its entire delta-v capability, regardless of the delta- $\mathrm{v}$ required to insert into the target orbit. The hybrid motor vehicle can cutoff its engine when commanded by guidance, preserving its excess energy in unspent propellant. The guidance strategy 
for each vehicle must respond to these inherent performance margin differences.

The original guidance concepts for the ascent vehicle employed a single, flexible guidance algorithm for both the solid motor or hybrid motor concepts. Subsequent analysis has demonstrated the need for independent guidance algorithms for each vehicle in order to account for both performance dispersions and available strategies for energy management. Since the computational capability of the avionics has yet to be analyzed, simple, robust guidance algorithms are prioritized in the current design whenever possible. The solid motor vehicle guidance is explained first, followed by the hybrid motor vehicle guidance.

\section{Solid Motor Vehicle Guidance}

The first stage solid motor vehicle guidance employs openloop guidance in which the vehicle commands a series of roll, pitch, and yaw angles from a pre-loaded lookup table as a function of vehicle altitude. The lookup table is derived from an optimized 3DOF trajectory using POST2 [4].

Second stage guidance for the solid motor vehicle employs SXS (Simple Cross-product Steering) [5]. This closed-loop guidance (CLG) algorithm is being developed at Marshall Space Flight Center (MSFC), and is based in cross-product steering (CPS) [7]. The algorithm is designed to target a desired semi-major axis and orbital plane, functioning without any inherent knowledge of motor performance. The output of SXS is a Vgo vector: the difference between the current vehicle's inertial velocity vector as estimated by navigation, and the inertial velocity vector calculated to achieve the semi-major axis and orbital plane of the guidance target. The simplicity of this algorithm allows it to function in a wide variety of off-nominal vehicle parameters.

The basic flow of SXS is as follows:

1. Load the RAAN, inclination, and semi-major axis of the target orbit (performed once per target set)

2. Load the current state as estimated by navigation

3. Calculate the unit vectors of the target orbit frame

4. Calculate the velocity-desired vector: the inertial velocity required to be on the target orbit plane, at the vehicle's current, estimated position, with an orbit whose Semi-Major Axis (SMA) matches the SMA of the target orbit

5. Calculate Vgo: velocity desired minus the current, estimated inertial velocity

6. Pass Vgo on to steering and controller functions

Neither open-loop or SXS are capable of managing excess energy in the solid motor vehicle. That task is performed by energy management. Given that the optimal location to perform an impulsive periapsis raise maneuver (PRM) is at the apoapsis of the current orbit [6] (or trajectory in this case), any impulsive delta-v maneuver not occurring at apoapsis will raise periapsis with a less efficient use of delta- $v$ than if the burn were performed at apoapsis. The solid motor vehicle energy management strategy takes advantage of this fact.
During coast, second stage guidance targets are loaded and closed-loop guidance calculations continuously occur. The magnitude of the Vgo vector output from guidance can be compared to a predicted onboard estimation of the delta- $v$ to be gained from the vehicle's second solid motor. As the vehicle coasts toward apoapsis, the delta-v required to achieve the target orbit decreases, while the predicted delta- $\mathrm{v}$ capability from the vehicle's upper stage changes only marginally in response to vehicle mass changes from RCS usage. Ignition of the second stage can then be commanded when the predicted onboard delta- $\mathrm{v}$ matches the required delta-v calculated by closed-loop guidance (Vgo).

This energy management strategy effectively shifts the time the second stage is ignited from apoapsis to some point prior to apoapsis in proportion to the amount of predicted excess delta-v capability onboard. The second burn is intentionally performed at a sub-optimal time in order to manage excess energy. The solid motors are sized so as to nominally impart more delta-v than required to meet the target orbit with known inert and payload masses, providing the vehicle with both the excess energy required to respond to off-nominal conditions and a guidance method to reduce the effect of those dispersions on orbital insertion accuracy.

The efficacy of this energy management architecture however, is heavily dependent on accurately predicting the delta-v capability of the second stage. This delta-v estimate can be pre-loaded either as a constant, or as an effective specific impulse and employ onboard mass-estimation to calculate the predicted delta-v. In either case, real-world deviations from these estimates translate into orbital insertion error.

In addition, it is worth noting that since this energy management architecture functions by altering the ignition time of the second stage, it cannot address variations in environment or performance that occur after second stage ignition.

Figures 1, 2, and 3 below show the results of a generic 6DOF simulation used to test the results of running the described guidance architecture on the upper stage of the vehicle. This sim was used to capture specific guidance sensitivities independently of the MANTISS simulation. In these runs, the 6DOF two-stage solid motor vehicle was dispersed by increasing the amount of total impulse delivered by stage one in each simulation run. The nominal vehicle contained more delta-v capability than was required to meet the target orbit nominally, and the vehicle targeted a known SMA and orbit plane. The runs were performed once with energy management active ('ON'), i.e. igniting the second stage when predicted delta- $v$ from stage two matches that required by CLG, and again with energy management inactive ('OFF'), i.e. igniting stage two at apoapsis. Figures 1 and 2 show that without energy management, the vehicle overshoots its target, causing an increased apoapsis and increasing SMA as the total impulse of stage one increases. 
In the plots, the blue points are clustered around the simulated target altitude conditions. With energy management active ('ON') however, the increasing apoapsis effect disappears and the increase in SMA caused by increasing stage one total impulse is minimized. Additionally, Figure 3 shows how the delta-time before apoapsis that stage two ignition occurs increases as the total impulse delivered from stage one increases. For the 'OFF' case, the burn time is fixed to occur at apoapsis.

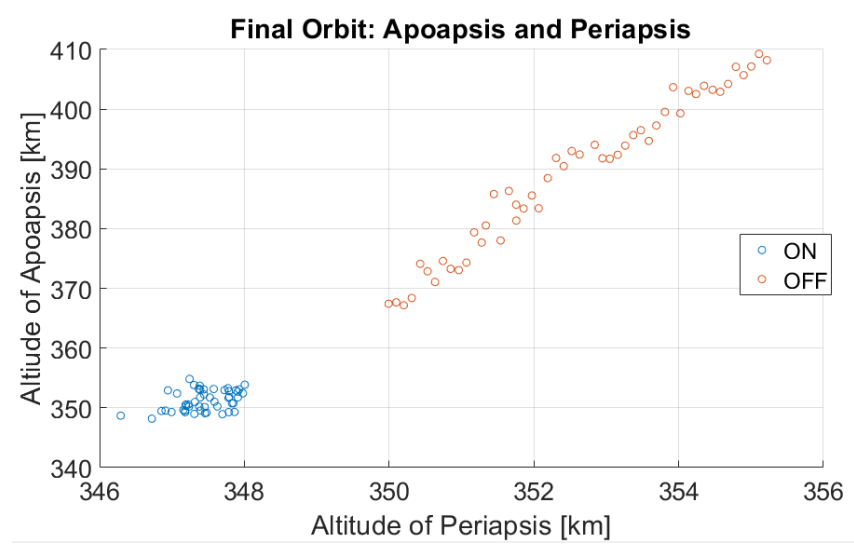

Figure 1: Effect of Energy Management on Orbit Apoapsis and Periapsis

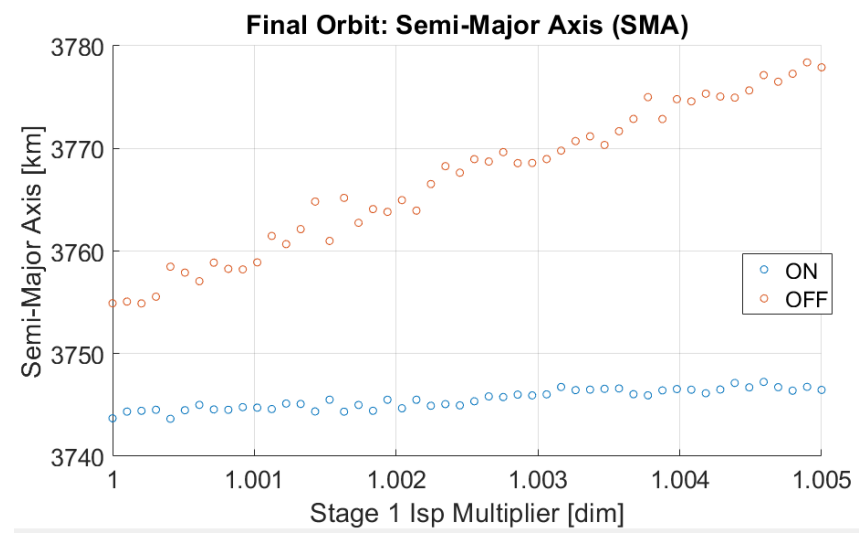

Figure 2: Effect of Energy Management on Semi-major Axis

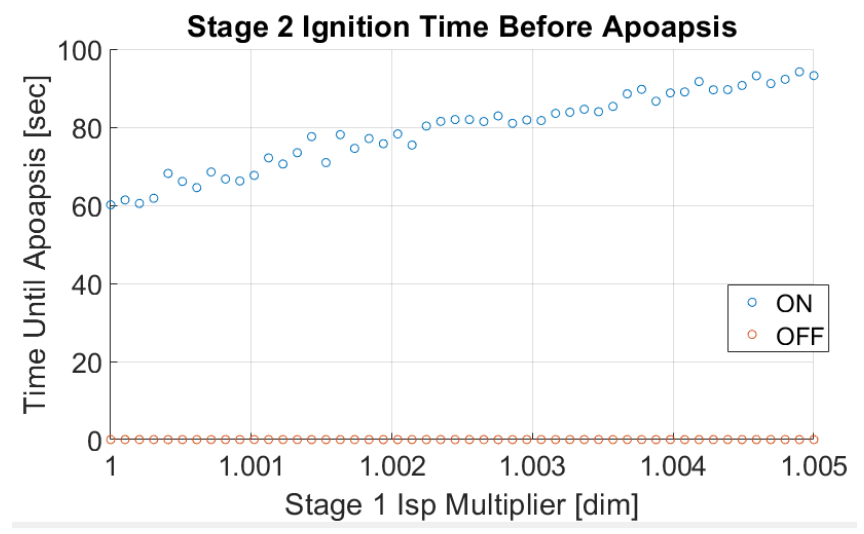

Figure 3: Effect of Total Impulse Correction on

\section{Second Stage Ignition Time}

Hybrid Motor Vehicle Guidance

The hybrid motor vehicle employs Powered Explicit Guidance (PEG) [8] for both burns. PEG is an explicit, pathadaptive, iterative guidance algorithm which assumes partialoptimality through applying the linear-tangent steering law. Since the hybrid motor vehicle contains the capability to command engine shutdown and provides a more constant thrust while burning, a wider number of conventional CLG algorithms were available. PEG has a history of successful flight implementation though the Shuttle program, making it a primary candidate for MAV guidance.

Although PEG does not explicitly account for atmospheric effects, the iterative nature allows PEG to correct for errors in vehicle stage that accumulate due to aerodynamic effects.

The current hybrid vehicle reference trajectory terminates the first burn during a state of non-negligible dynamic pressure. The current vehicle center of mass, center of pressure, and reaction control system thruster locations increase the need to address aerodynamic torques in order to maintain controllability at the end of the first burn. This is accomplished by switching from PEG to an aerodynamic angle nulling guidance at a predetermined time before the PEG-predicted cutoff time of the first burn. This second guidance algorithm uses the current vehicle body orientation, as estimated by navigation, to estimate the aerodynamic angles, and steers to ramp the angles to zero over a 5-second period. Guidance then continues to command zero aerodynamic angles as the vehicle coasts through the remainder of the appreciable dynamic pressure. This approach minimizes the magnitudes of the aerodynamic torques in order to maintain body orientation at burnout in a vehicle potentially capable of marginal stability configurations.

The second burn is initiated at apoapsis, and the engine commanded to shutdown when the vehicle has achieved the target orbit within guidance tolerances. Excess energy remaining is in the form of unspent propellant.

\section{NAVigation Design AnALYSIS}

The capability of the above discussed guidance algorithms to meet orbital insertion performance is inherently limited by the ability of the vehicle to track its own state. Due to the lack of external infrastructure, the navigation solution must rely o a purely inertial solution. This is achieved through the measurement of inertial acceleration and angular rate in the sensor frame (rotated with some uncertainty to the body frame for controls) of the vehicle. To propagate the state forward over the ascent trajectory, a $2^{\text {nd }}$ order high rate state integration process is used to track the vehicle attitude to a given inertial frame, rotate sensor-frame accelerations into the inertial frame, estimate the local gravitational force, and propagate the onboard state forward. 
In order to assess the capability and performance of the navigation systems the detailed IMU sensor model developed in [1] was integrated into the simulation framework. A block diagram of the implementation is given below in Figure 4. This model takes the true inertial total accelerations, removes the gravity components, and transforms them into the body frame. This model also includes a star tracker model implementation, which uses body frame inertial acceleration and angular rates to provide constraints on operations (the results of this functionality are discussed in the final subsection). The IMU model will then apply a variety of errors to the truth in order to provide a sensed measurement. These individual errors are dispersed and enabled via input files mechanism with an automated approach to one-at-atime sensitivities and analysis.

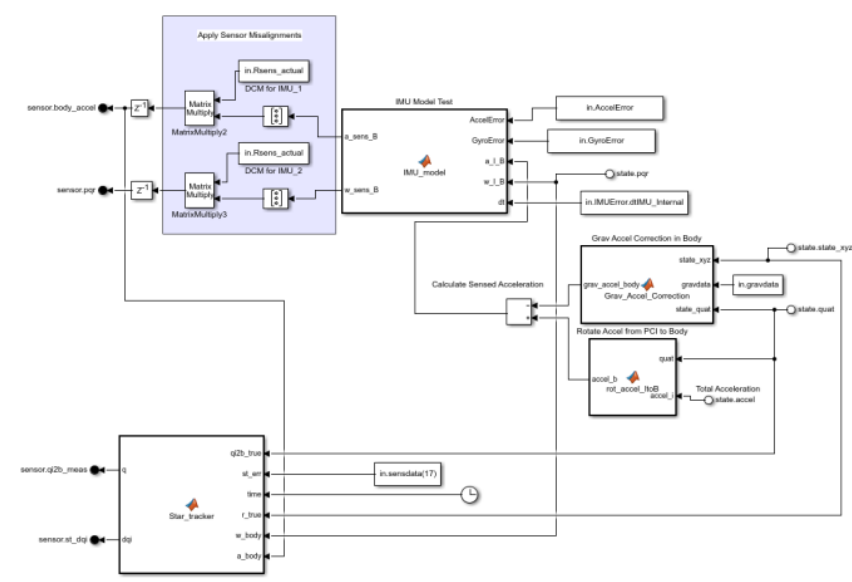

Figure 4: Sensor Model Architecture

While IMU errors drive a large amount of navigation errors along the trajectory, much of this is dependent on the initial state knowledge of the vehicle (time, position, velocity, and attitude). In order to define requirements for state initialization, these terms are modeled in the simulation as an external measurement, rather than an internally calculated value (by a method such as gyrcompassing). The focus is on determining system requirements on initial knowledge. This input is provided as an uncertainty within the input file, allowing for ease of trade studies and sensitivity analysis.

In terms of IMUs, the state of the art exhibits a strong correlation between performance and SWAP (Size, Weight, and Power). In order to achieve an accurate insertion state, a navigation grade unit is typically required, consuming a large amount of physical space. But the constraints on the integrated system directly place stringent limitations on the available SWAP for the IMU. This behavior can be observed in Figure 5, which depicts three potential navigation platforms (tactical MEMS - STIM300, low-grade navigation HG5700, and high-grade navigation MIMU platforms) to scale in terms of total enclosed volume. Power required and mass show similar trades, syncing performance to physical characteristics.

As such, the analysis initially focused on a range of options that are discussed herein. The baseline design was selected to be a HG5700 unit, a new product coming online in 2019 from Honeywell. This was selected due to its superior performance over the HG1700, providing a low-grade navigation capability within a small mass and volume with some flight heritage. This was used to capture a potential option in terms of performance capability, and provided adequate performance within a compact package. Elements such as environmental and operational qualification are discussed later in this section in discussion of path to flight.
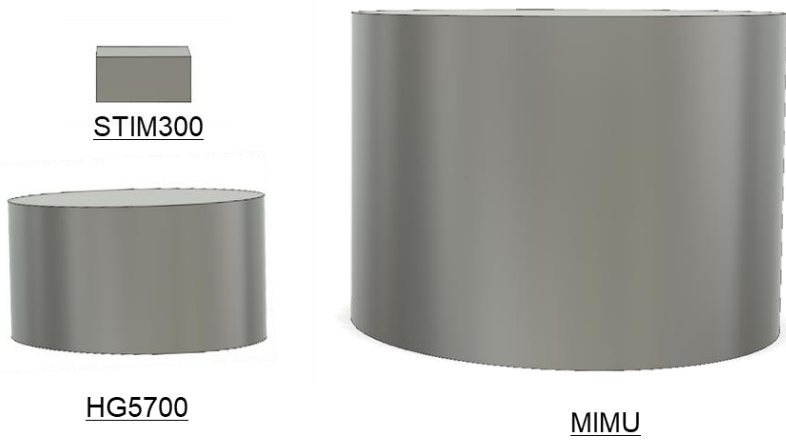

Figure 5: Comparable Volume of Potential IMUs

\section{Navigation Capability for Solid Vehicle}

In order to provide insight to the integrated performance of the system, Monte Carlo analysis was used to assess the vehicle's integrated insertion capability in terms of altitudes of periapsis and apoapsis. These two metrics provide insight into the in-plane and orbital shape errors for the target orbit. Using the full simulation environment allows for inclusion of nonlinear sensitivities in the interaction of guidance, navigation, and control algorithms across the vehicle. The results here focus on the capability using the solid vehicle. While flying a slightly longer trajectory, similar trends were observed with the hybrid configuration and thus are not included in this documentation.

The first set of cases focus on assessing the performance with the baseline navigation system for a notional orbital target. For this scenario, 1000 cases were assessed, each assuming perfect initialization knowledge with dispersed sensor error terms derived from the vendor specifications. Figure 6 shows the final insertion conditions of the vehicle. As seen, due to the short duration of the trajectory, with perfect initial knowledge the actual inserted states are very close to the result of the navigation system. In these plots, the blue dots represent the actual final state and the red captures the navigated states (onboard knowledge). The difference in slope between the navigated and truth states is likely due to integrated attitude errors (through gyro bias) interacting with the guidance algorithms. The small overall errors here though show minimal impact to performance.

As initial state knowledge errors are included, these dispersions quickly grow to larger levels. This is shown in Figure 7. With this degradation in initial knowledge, the 
vehicle's navigation state errors continue to grow, and the actual inserted states show a much larger dispersions. For example, as seen in the plots, the variation in inserted altitude at apoapsis essentially doubles in breadth. The similar distribution of the navigated states in both scenarios show the guidance algorithms driving the vehicle to a similar expected insertion state. The dispersions here on the actual inserted conditions are due online to navigation effects. All other dispersions were disabled in this analysis.

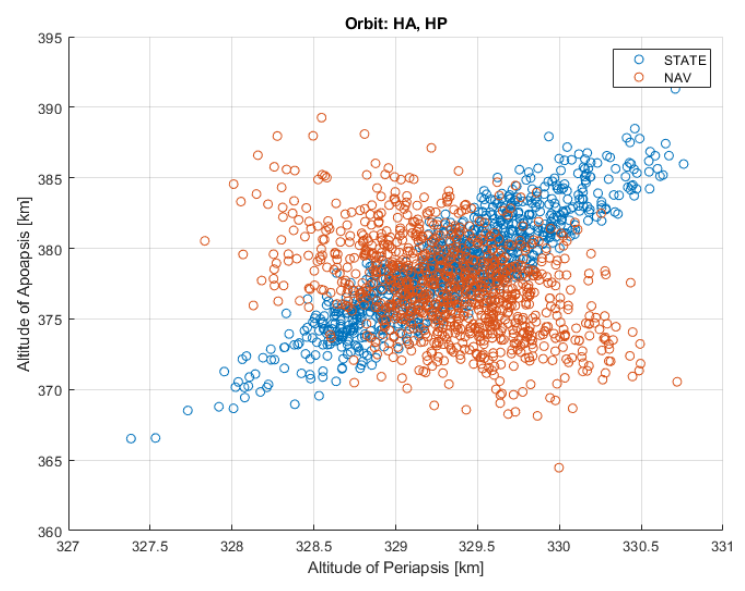

Figure 6: Effect of IMU Errors with Perfect Initial State Knowledge

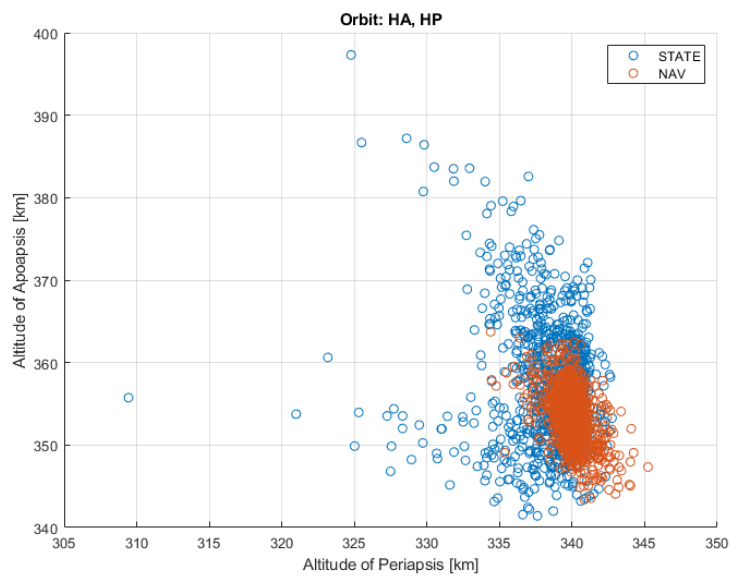

Figure 7: Effect of IMU Errors and Uncertainty in Initial State Knowledge

\section{Impact of initial errors}

In order to provide more insight into the sensitivity to these initial errors on the final solution, a detailed analysis was performed to identify key drivers. This effect is clearly seen in Figure 8, which shows the effect of initial knowledge uncertainties only (assuming a perfect IMU). The relationship here shows that increases in initial errors cause growth in orbital insertion shape. The bimodal behavior seen here is a consequence of initial azimuth errors being amplified via their large effect on the final orbit shape. Not shown, but similarly, these uncertainties in initial conditions additional cause out-of-plane errors, affecting the vehicle's inclination and right ascension of the ascending node. The
IMU-induced errors blur the resulting shape since in this chart as they can effectively "counteract" initial attitude errors over flight in some scenarios. It is important for the MAV to limit both in- and out-of-plane dispersions in order to meet the capability of the orbital element that will rendezvous and capture the samples that are being placed into orbit.

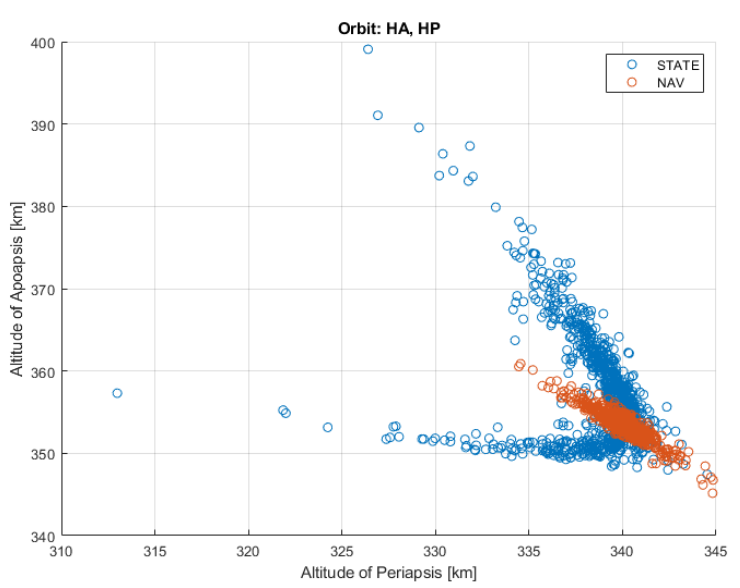

Figure 8: Effect of Initial Knowledge Errors Only

A sensitivity analysis was used to capture the key drivers on insertion performance. A Monte Carlo-based variance technique was used to capture individual parameter sensitivities. In this analysis, a Monte Carlo was performed with all sensor terms dispersed to capture total output variance. Then individual terms (or group of terms) were enabled to capture variance on the output due to the variance on those input specific input parameters. The fraction of the variance due to a specific term over the total variance gives an approximate understanding of the sensitivity to that parameter. This analysis focused on breaking down the sensitivity on insertion accuracy based on attitude, position, and velocity initial variance as well as IMU error dispersions.

The results of the analysis are given in Figure 9 and Figure 10 below. In each of these, it can be seen that the initial position and velocity dispersions (at the level analyzed, 100 meters, and .1 meters/second one-sigma) were a minor contributor, their primary effect being in providing an inaccurate state for gravity calculations.

The primary driver in both cases is shown to be the initial attitude errors. This pointing error directly reduces the accuracy of the open-loop first stage maneuver on launch from the lander. Due to the large amount of DV being imparted over this stage, the dispersions on the state at the end of the burn are increased. Similarly, this initial attitude error persists throughout the simulation, causing increased attitude errors, but also errors in translating sensor frame accelerations into the initial frame, causing an increase in translational errors. Additionally, this attitude error limits the accuracy of pointing maneuvers commanded from closedloop steering. IMU-errors are a secondary driver and have a 
larger effect on periapsis than apoapsis. In terms of other orbital elements, semi-major axis, true anomaly, and argument of periapsis variation behaved similarly to that shown in Figure 9, while Eccentricity and Inclination are similar to Figure 10 with initial attitude error being an larger system driver. Note that the accuracy of sensitivity analysis is limited by the number of cases run and linear variance assumptions in the sensitivity analysis approach.

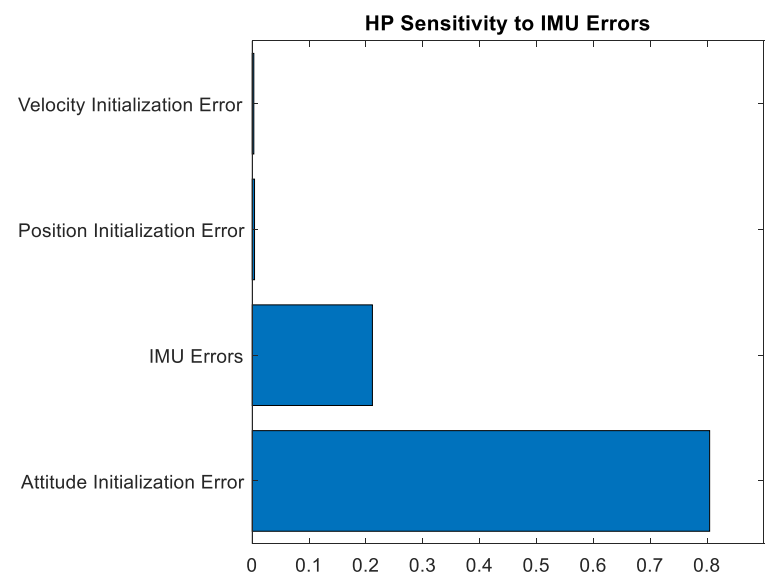

Figure 9: HP Sensitivity to Navigation Errors

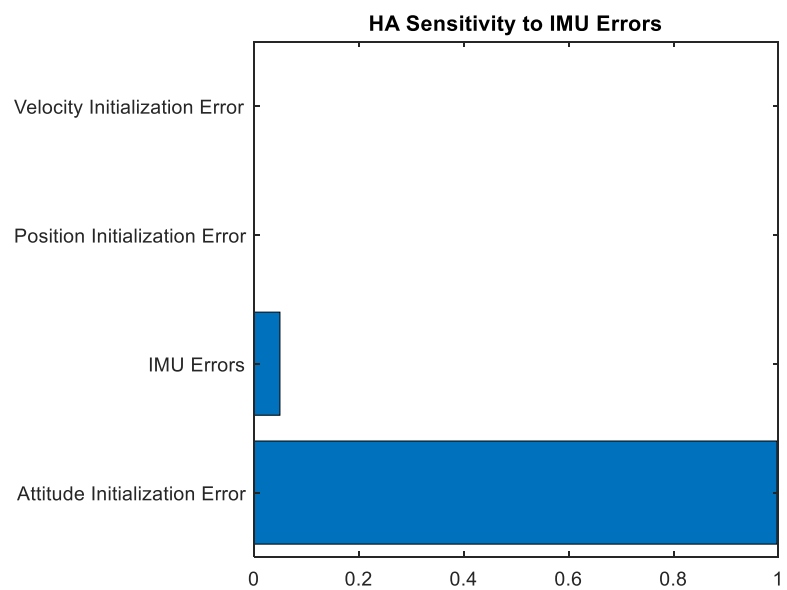

Figure 10: HA Sensitivity to Navigation Errors

\section{Individual Sensor Error Sensitivities}

With this analysis, the next step is to understand individual sensor error terms in order to determine primary drivers in IMU selection. To start this analysis and identify the key variables, another variance-based sensitivity analysis was performed. In these simulations, individual sensor terms were enabled to capture primary drivers. Included in this study for the accelerometers and gyroscopes are terms such as turn-on bias, scale factor, non-orthogonality, internal misalignments, and random walk terms. For each error input term, a 500 case Monte Carlo was performed to get insight into sensitivities.

The results of this analysis are given in Figure 11 and Figure
12 to match the primary results shown in this paper, focused on altitude of periapsis and apoapsis. From this analysis, the accelerometer bias in the body- $\mathrm{x}$ axis is the most sensitive parameter to both of these terms. This is due to the term being in the thrust direction of the vehicle. Due to the orbital shape, this in-plane bias directly affects the final orbit shape achieved. Similarly, the second driving term is accelerometer scale factor in the body $x$ direction. Again, the alignment of this error term with the thrust axis of the vehicle results in a scaling of the observed applied acceleration. Thus scaling results in integrating too much or too little thrust, and with the large thrusts imparted on the vehicle results in larger insertion errors. Tertiary effects include gyro biases and scale factor terms, and the effect of integrated attitude errors on the translational state is clear.

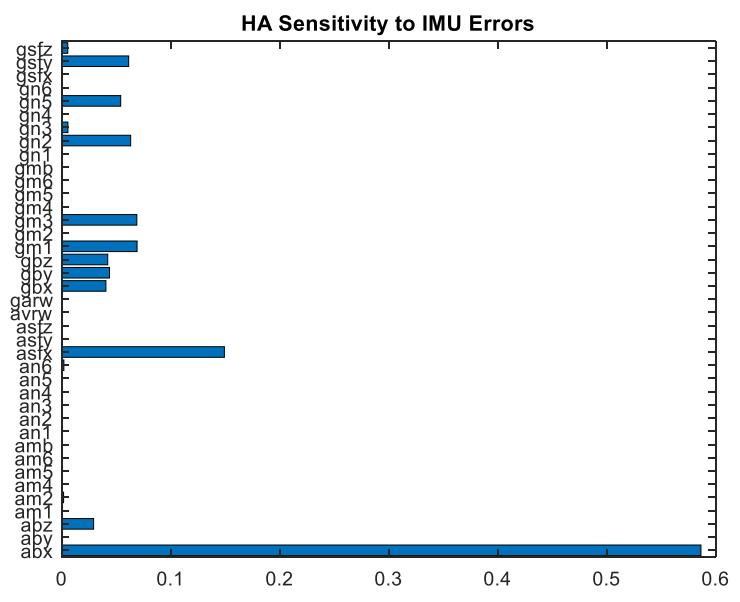

Figure 11: HA Sensitivity to Individual IMU Errors

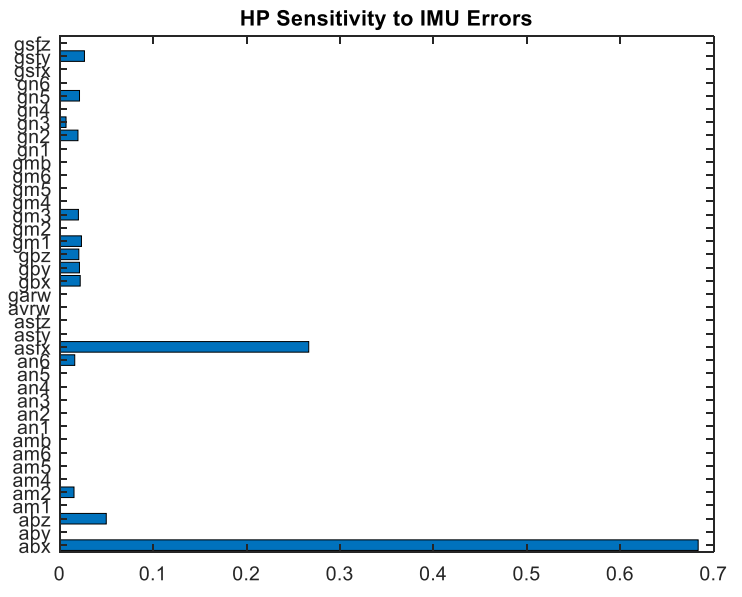

Figure 12: HP Sensitivity to Individual IMU Errors

To help define operational constraints on accelerometer and gyroscope performance (as well as driving potential gyrocompassing capability), a series of 1-d analysis trades were performed to assess insertion performance as a function sensor bias terms. For each, the values were dispersed across a linear range and a simulation for each was run with all other dispersions turned off. This allows insight into expected 
simulation behavior and high level trends.

For gyroscope bias errors, all axis primarily affected the phasing of the orbit and its out-of-plane characteristics (i.e. off-target inclination). The simulations showed minor effects on the final orbital shape and altitude, showing the tertiary effect of these terms as identified in the previous sensitivity analysis. Two of these plots are given in Figure 13. This identifies the strong correlation between uncertainties in bias about the body z-axis (equating to a vehicle roll).
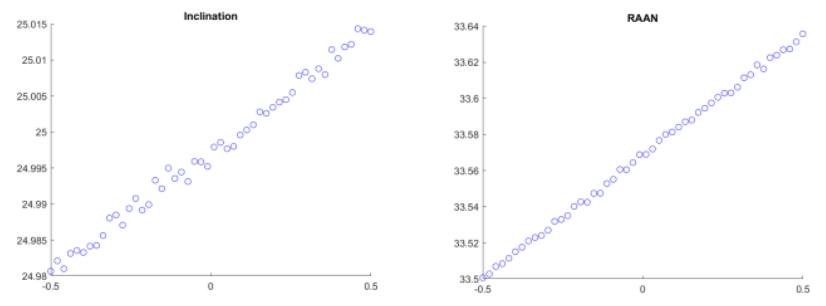

Figure 13: Out-of-plane Error Sensitivities in Inclination (deg) (L) and Right Ascension of the Ascending Node (deg) (R) to Gyro Bias in Body-Z (deg/hr)

Similar analysis was performed to capture the 1 dimensional sensitivity to accelerometer bias uncertainty. As the primary IMU sensitivity to altitude of periapsis and apoapsis, this analysis can provide insight to sensor selection for required ascent performance. Again, the accelerometer bias in each body axis vas varied across a range of values and simulations were run with only that error dispersed. Some of the results of this analysis are provided in Figures 14 -16. Each of these plots shows insertion performance against magnitude of accelerometer bias in Earth-g's. The displayed divergent behavior is due to the modeling of positive and negative biases.

Some of the more interesting results are the sensitivity across each axis to the shape and phasing of the orbit. For example, off-axis accelerometer biases exhibit a large effect on the apoapsis errors for $\mathrm{x}$-axis errors (typically pointing along the orbit plane and primarily affect the shape of the orbit. This is shown in Figure 14. In contrast, accelerometer bias in the other lateral direction (aligned out of the target plane), exhibit a larger effect on the orbital plane alignment, i.e. inclination and right ascension of the ascending node. Figure 15 shows this behavior. The largest driver to orbital shape though is the accelerometer bias along the body's z-axis, aligned with thrust. Errors in this axis also contribute to in-plane errors, with a strong effect on energy attained, semi-major axis, and orbit eccentricity. This sensitivity shows that for accelerometer biases above $.01 \mathrm{~g}$ 's, the insertion performance quickly degrades away from the requirement.

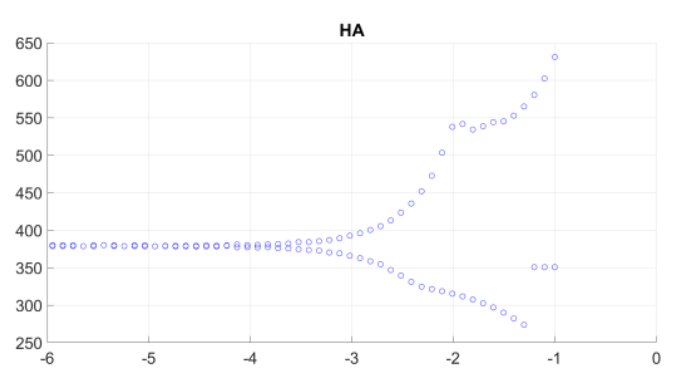

Figure 14: HA (km) as a function of Accelerometer Bias in Body-X $(\log \mathrm{g})$

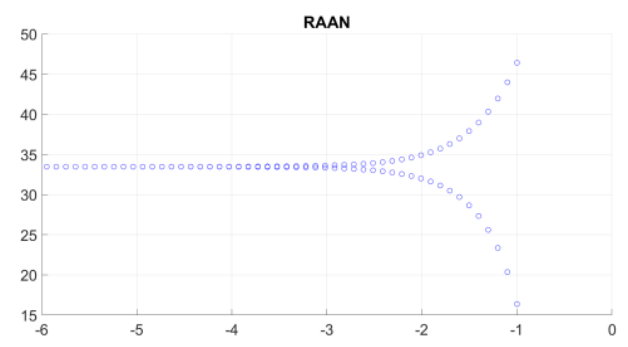

Figure 15: RAAN (deg) as a Function of Accelerometer bias in Body Y (log g)
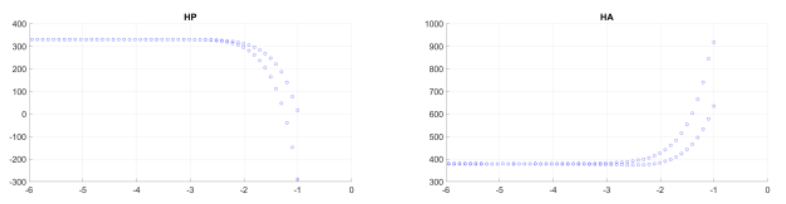

Figure 16: Orbit Shape (km) as Function of Accelerometer Bias in Body Z $(\log \mathrm{g})$

\section{Integration of Star Tracker}

In order to reduce attitude errors prior to the second bun maneuver, which is critical to entering into the desired orbital shape), the team assessed the performance gained by including a star tracker on the ascent vehicle. For this analysis, the additional sensor was assumed to provide an inertial attitude solution with 100 arcseconds one-sigma accuracy with a mounting uncertainty of $1 / 8$ degree. Operational constraints were implemented into the simulation to limit operation based on altitude, acceleration, and angular rate. Thus the sensor would only provide valid operations at high altitudes (ensuring a clear view of the sky) when the vehicle was coasting (low rates and applied acceleration). With an input measurement, the new attitude was taken as a direct update to the integrated attitude position due to its vastly increased accuracy over the inertial solution. Further performance could be gained through the implementation of a filter to smooth and integrate the measurements.

The goal of this analysis was to assess the potential for inclusion of a lower grade, much lighter sensor (the STIM300) for inclusion on the vehicle. Figure 17 presents the nominal performance of the inertial only solid vehicle under navigation dispersions. As seen, while the vast majority of 
the baseline sensor cases fall within the desired altitude band, the STIM exhibits a much larger dispersion. With the inclusion of the star tracker as seen in Figure 18, this is significantly reduced with $71 \%$ of cases (up from 17\%) meeting the desired performance. This shows potential for inclusion if the insertion error requirements can be opened up at the architectural level. For the baseline, the star tracker is being removed due to the operational constraints imposed by the vehicle, including attitude maneuvering for a clear view of the sky and the potential for dust buildup on the lens.

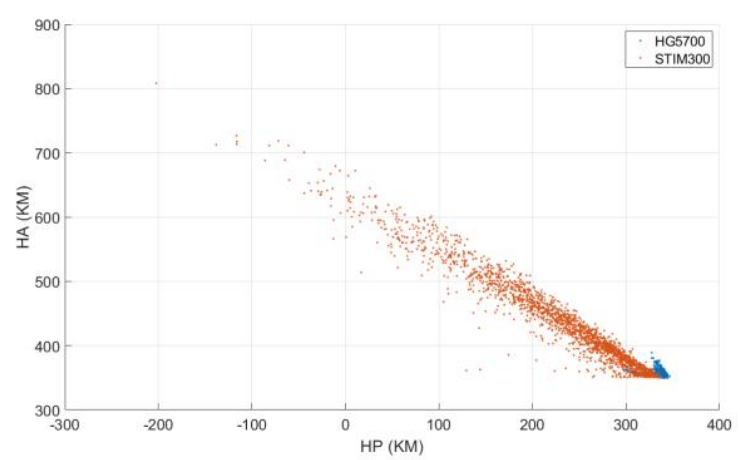

Figure 17: Inertial Only Results (0.1 Deg. Attitude Knowledge) (0.2 STIM: 17\% HG5700: 99.5\% Success)

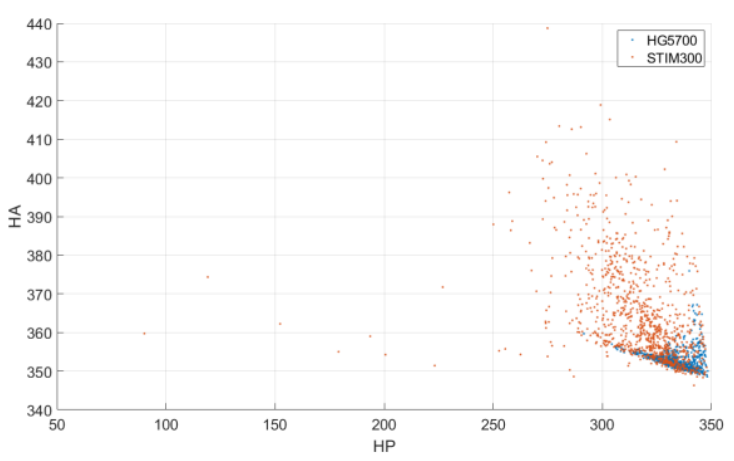

Figure 18: Star Tracker-enabled Improvements (0.1 Deg. Attitude Knowledge) (STIM: 71.3\% HG5700: 99.8\%)

\section{Integrated Vehicle Performance}

In order to better understand off-nominal vehicle performance, 6DOF simulations were run across a range of dispersed parameters in both 1-dimensional sensitivities in which a single parameter was varied across a known range, and in Monte Carlo analysis, in which all parameters were varied randomly with each seed according to their respective, statistically-assumed bounds. Table 1 summarizes the parameters varied in the Monte Carlo analysis of the solid motor vehicle.

Solid motor variations were performed through scaling equations applied separately to each stage. Atmospheric dispersions were performed by both varying the inputs to
Mars-GRAM, and utilizing the inherent dispersion capability within Mars-GRAM to disperse atmospheric values and winds.

Table 1: Dispersed Parameters

\begin{tabular}{|c|c|}
\hline Parameter & Unit \\
\hline Launch Elevation & {$[\mathrm{deg}]$} \\
\hline Launch Azimuth & {$[\mathrm{deg}]$} \\
\hline Launch Site Position: X,Y & {$[\mathrm{m}]$} \\
\hline Launch Site Position: Z & {$[\mathrm{m}]$} \\
\hline Vehicle Mass Variation & {$[\mathrm{kg}]$} \\
\hline ST1 Moments of Inertia & {$[\%]$} \\
\hline ST2 Moments of Inertia & {$[\%]$} \\
\hline ST1 CG Offset: X,Y,Z & {$[\mathrm{m}]$} \\
\hline ST2 CG Offset: X,Y,Z & {$[\mathrm{m}]$} \\
\hline Aerodynamic Coefficients & {$[\%]$} \\
\hline Atmospheric Conditions & {$[\mathrm{dim}]$} \\
\hline Sensor Position Knowledge Error: X,Y,Z & {$[\mathrm{m}]$} \\
\hline Sensor Initial Attitude Knowledge Error & {$[\mathrm{deg}]$} \\
\hline Sensor Orientation Error & {$[\mathrm{deg}]$} \\
\hline SRM 1 Impulse & {$[\%]$} \\
\hline SRM 1 Burn Rate & {$[\%]$} \\
\hline SRM 1 Trace Shape & {$[\%]$} \\
\hline SRM 2 Impulse & {$[\%]$} \\
\hline SRM 2 Burn Rate & {$[\%]$} \\
\hline SRM 2 Trace Shape & {$[\%]$} \\
\hline SRM 1 Thrust Misalignment & {$[\mathrm{deg}]$} \\
\hline SRM 2 Thrust Misalignment & {$[\mathrm{deg}]$} \\
\hline
\end{tabular}

Monte Carlo Analysis Results

Figures 19-23 below show the results of a 2000 seed Monte Carlo analysis dispersing the parameters outlined in Table 1 above. For each run a random value with the bounds was selected for each dispersed parameter according to its predicted statistical distribution. The 6DOF simulation results were then compiled for all 2000 seeds.

Figures 19-23 show that under the current dispersion bounds, the solid motor vehicle 6DOF final orbital states are within the current bounds of semi-major axis and eccentricity, and no points violate the minimum periapsis constraint. Variation in final orbit inclination is within the current bounds. It is worth noting that at the time of this publication, the bounds on acceptable final orbit dispersion in RAAN were in flux. Though these results and plots are part of ongoing work, they demonstrate the feasibility of the current solid motor design to respond to dispersions and approach target orbit accuracy goals. 


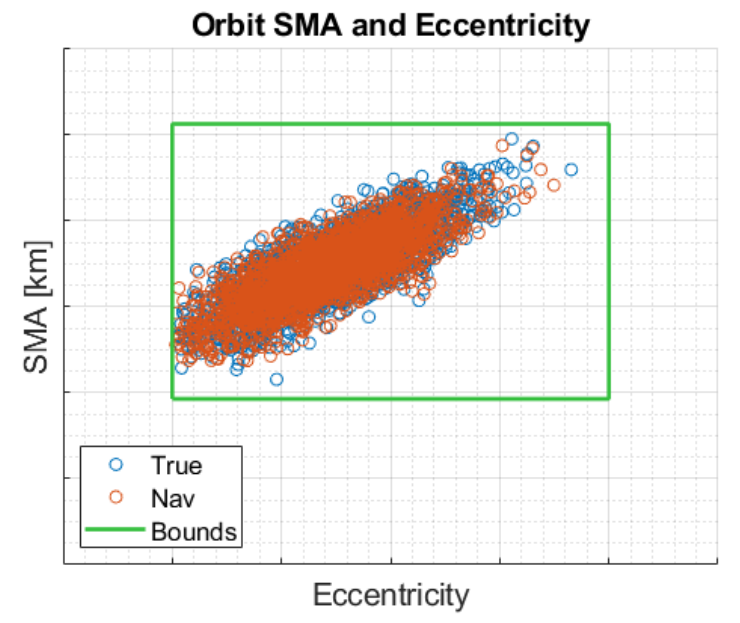

Figure 19: Monte Carlo Orbit Results: SMA and Eccentricity Orbit Apoapsis and Periapsis

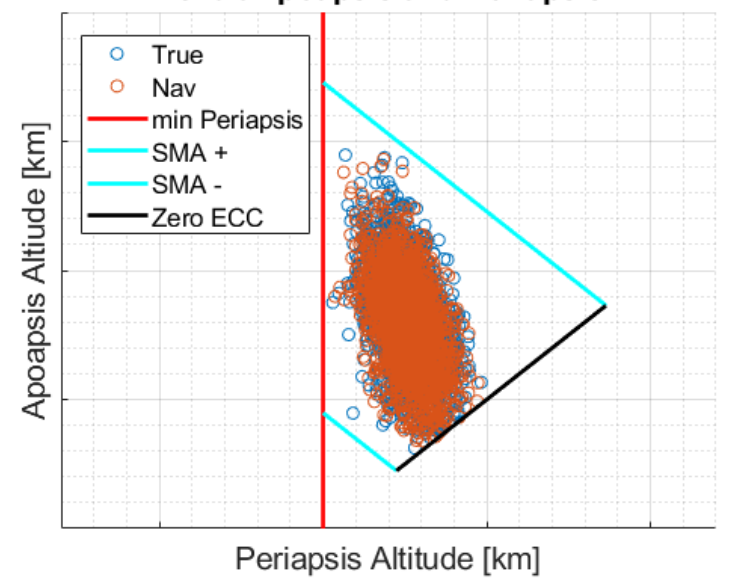

Figure 20: Monte Carlo Orbit Results: Periapsis and Apoapsis

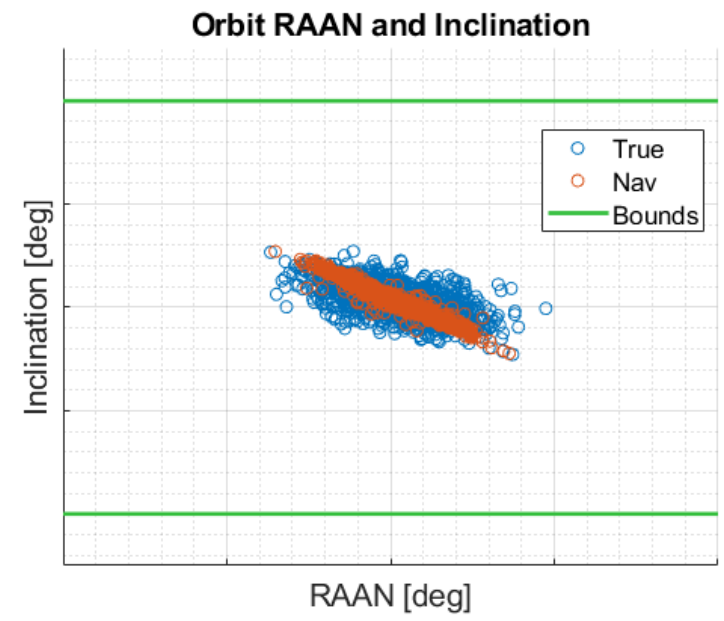

Figure 21: Monte Carlo Orbit Results: RAAN and Inclination

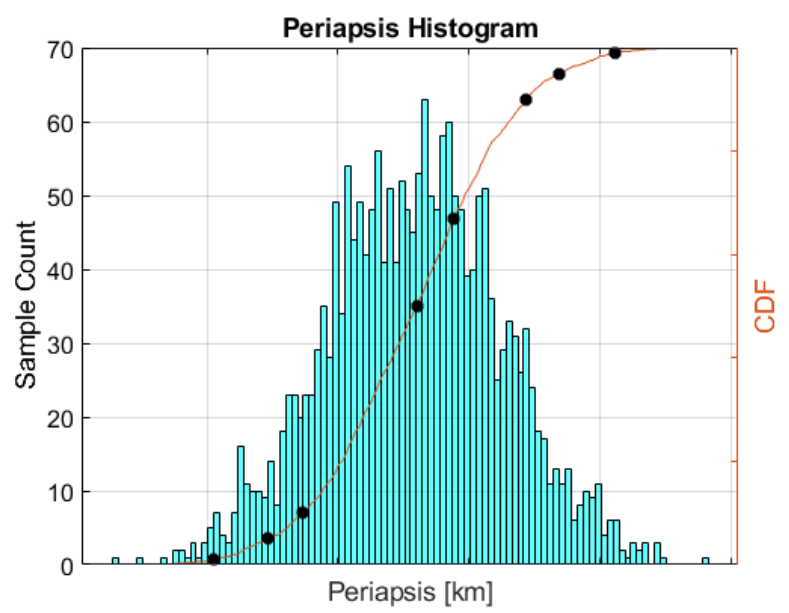

Figure 22: Monte Carlo Orbit Results: Periapsis Histogram

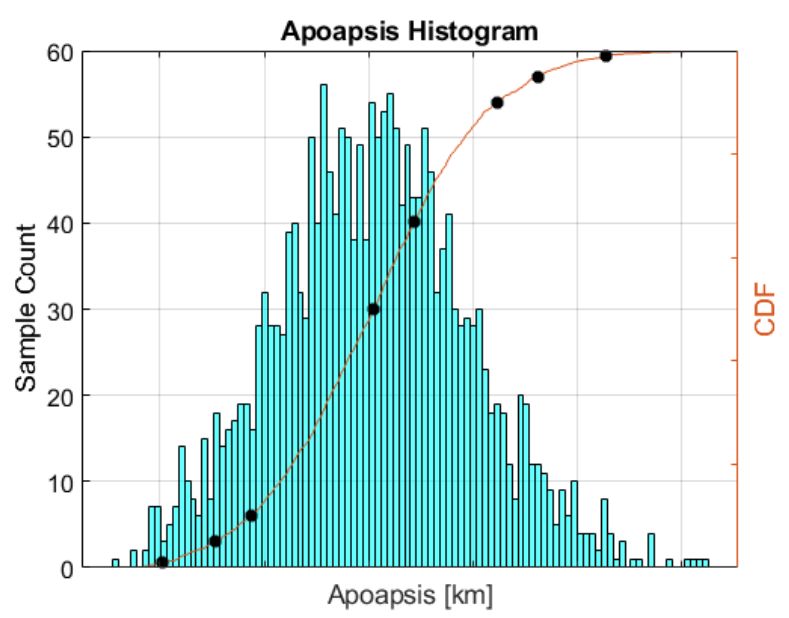

Figure 23: Monte Carlo Orbit Results: Apoapsis Histogram

Similar analysis for the hybrid motor vehicle is currently underway.

1D Sensitivity Analysis

In addition to Monte Carlo analysis, the individual effects of dispersing a single parameter on orbit insertion accuracy were assessed. A subset of the parameters selected for the Monte Carlo analysis were used, and the parameter was varied linearly across the range indicated in the first three columns of Table 2. This range is not the same range that was used for the Monte Carlo analysis. The 1D sensitivities intentionally varied each parameter beyond its anticipated bounds in order to capture any outlying edge behavior. For each run, a single parameter was varied. The table identifies mass as the total mass of the vehicle at launch independently of uncertainties in mass changes due to engine burn parameters. For example, one scenario can be additional mass on the payload outside of expectations (i.e. extra dust or mass of samples). The last two columns in Table 2 summarize the 
results by showing the semi-major axis effects observed for the solid motor vehicle for both truth and navigation states.

Table 2: 1-D Sensitivity Summary

\begin{tabular}{|c|c|c|c|c|c|}
\hline $\begin{array}{c}\text { Dispersed } \\
\text { Parameter }\end{array}$ & Range & Unit & $\begin{array}{c}\text { True State } \\
\text { SMA Range: } \\
\text { Deviation } \\
\text { from Nominal } \\
{[\mathbf{k m}]}\end{array}$ & $\begin{array}{c}\text { Navigation } \\
\text { State SMA } \\
\text { Range: } \\
\text { Deviation from } \\
\text { Nominal } \\
{[\mathbf{k m}]}\end{array}$ \\
\hline $\begin{array}{c}\text { Axial X CG } \\
\text { Offset }\end{array}$ & \pm & 0.01 & {$[\mathrm{~m}]$} & 1.56 & 1.22 \\
\hline $\begin{array}{c}\text { Lateral YCG } \\
\text { Offset }\end{array}$ & \pm & 0.01 & {$[\mathrm{~m}]$} & 2.95 & 1.96 \\
\hline $\begin{array}{c}\text { Lateral Z CG } \\
\text { Offset }\end{array}$ & \pm & 0.01 & {$[\mathrm{~m}]$} & 8.34 & 7.40 \\
\hline Mass & \pm & 1 & {$[\mathrm{~kg}]$} & 91.04 & 91.03 \\
\hline Stage 1 Impulse & \pm & 1 & {$[\%]$} & 6.99 & 7.21 \\
\hline $\begin{array}{c}\text { Stage 1 Burn } \\
\text { Rate }\end{array}$ & \pm & 10 & {$[\%]$} & 3.07 & 2.42 \\
\hline $\begin{array}{c}\text { Stage 1 Thrust } \\
\text { Misalignment Y }\end{array}$ & \pm & 1 & {$[\mathrm{deg}]$} & 9.76 & 9.56 \\
\hline $\begin{array}{c}\text { Stage 1 Thrust } \\
\text { Misalignment Z }\end{array}$ & \pm & 1 & {$[\mathrm{deg}]$} & 1.71 & 1.91 \\
\hline Stage 2 Impulse & \pm & 1 & {$[\%]$} & 75.48 & 75.20 \\
\hline $\begin{array}{c}\text { Stage 2 Burn } \\
\text { Rate }\end{array}$ & \pm & 10 & {$[\%]$} & 8.80 & 8.54 \\
\hline $\begin{array}{c}\text { Stage 2 Thrust } \\
\text { Misalignment Y }\end{array}$ & \pm & 1 & {$[\mathrm{deg}]$} & 0.39 & 0.41 \\
\hline $\begin{array}{c}\text { Stage 2 Thrust } \\
\text { Misalignment Z }\end{array}$ & \pm & 1 & {$[\mathrm{deg}]$} & 0.20 & 0.62 \\
\hline
\end{tabular}

By examining Table 2, it can be seen that the primary drivers for semi-major axis orbit accuracy for the solid motor vehicle among those examined are known vehicle mass and second stage total impulse. These two parameters both demonstrate a direct correlation to SMA deviation from nominal and a more significant magnitude of SMA deviation from nominal than the other parameters shown. Given the discussion on the implementation method of SXS and energy management above, this behavior is expected. Unknown variations in vehicle mass or second stage impulse both translate directly to variation in second stage delivered delta-v. As noted earlier, energy management is not capable of addressing dispersions in second stage delta-v. In terms of mass, while the vehicle mass itself will be known under assembly, limited capability is currently included to assess the actual weight of samples as loaded. The intention of the analysis is to identify the need to accurately understand vehicle mass at launch to inform mass estimation and guidance algorithms.

Similar 1-dimensional analysis for the hybrid motor vehicle is currently underway.

\section{Sensitivity Analysis and Key System Drivers}

To provide insight into the primary sensitivities of the integrated vehicle systems, including all dispersion effects currently modeled, a variance based approach was used to assess contributions to insertion errors. Again, this approach used a Monte Carlo technique and processed the results of one-at-a-time dispersions to assess contributions to total variance of the system. For this analysis, the dispersions were broken down into several categories: Initial Conditions (truth location and orientation at launch), Mass (uncertainties in payload and vehicle mass), Initial Knowledge (uncertainties in onboard position and attitude), Thrust (capturing dispersions in ISP and thrust profile), Aerodynamics (uncertainty in aerodynamic properties and wind dispersions), and IMU (capturing the effect of sensor errors). For each case, 500 runs were performed to get a high-level view of these. For this analysis, the baseline assumptions for initial knowledge and IMU specification were used. The summary of the results are given in Table 3 below.

Two of the more interesting results are in terms of the orbital shape sensitivities and inclination are given in Figure 24 and 25 . For the semi-major axis, a relative measurement of the total energy in the orbit, uncertainty in thrust and mass highly correlate to the ability to meet an inserted orbital energy. As the onboard knowledge of the mass is incorrect, the propagations of the state forward in time in assessing thrust applied to the stage are also incorrect, resulting in an wide dispersion of achieved orbits. Similarly, uncertainty in thrust directly affects the ability of closed-loop guidance to propagate the state forward and steer the vehicle. Similarly, increased dispersions in total impulse and thrust shaping on the second stage directly relate to excess (or loss) of energy put into the vehicle outside of expectations. While first stage guidance via energy management can correct for uncertainties in the first stage, this remaining dispersion directly correlates to orbital dispersions.

Table 3: MC Sensitivity Results (variance/total variance)

\begin{tabular}{|l|c|l|c|c|c|}
\hline & HA & HP & Inclin. & RAAN & SMA \\
\hline $\begin{array}{l}\text { Initial } \\
\text { Conditions }\end{array}$ & 0.00 & 0.00 & 0.00 & 0.00 & 0.00 \\
\hline Mass & 0.35 & 0.20 & 0.11 & 0.04 & 0.36 \\
\hline $\begin{array}{l}\text { Initial } \\
\text { Knowledge }\end{array}$ & 0.12 & 0.04 & 0.40 & 0.54 & 0.10 \\
\hline Thrust & 0.44 & 0.56 & 0.21 & 0.08 & 0.41 \\
\hline Aerodynamics & 0.06 & 0.32 & 0.14 & 0.05 & 0.00 \\
\hline IMU & 0.04 & 0.01 & 0.01 & 0.03 & 0.06 \\
\hline
\end{tabular}


Conversely, the primary effect on dispersed inclination comes from initial errors. This ties very closely to the initial attitude knowledge, particularly about the local azimuth direction at launch. Errors in this attitude correlate into outof-plane errors and drive the vehicle to an incorrect inclination. The other dispersions still contribute, but to a lesser degree, showing the impact of thrusting out of plane.

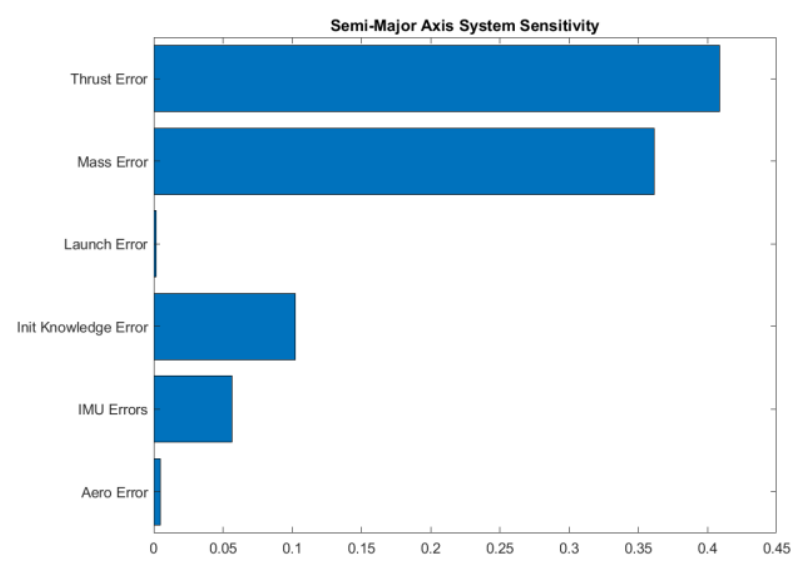

Figure 24: Semi-Major Axis Sensitivity

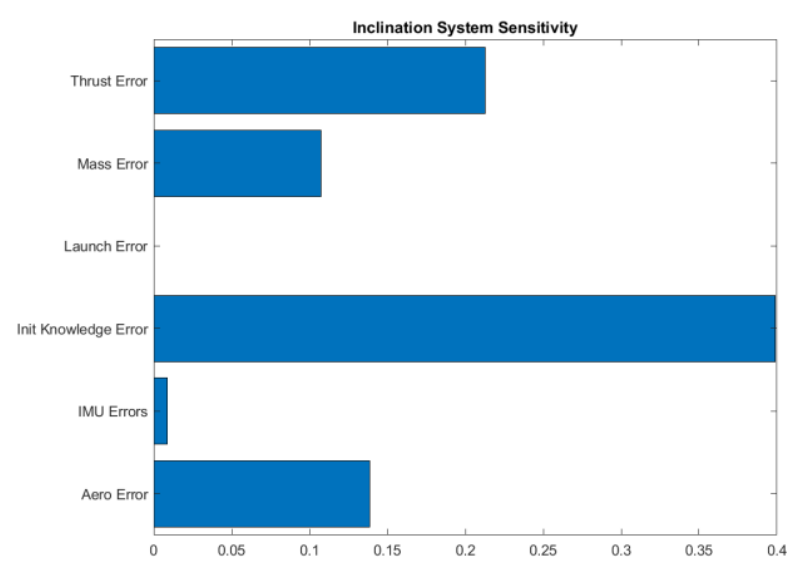

Figure 25: Inclination Sensitivity

\section{Impact of Navigation Grade IMU}

As mentioned the baseline IMU was selected as an aggressive option to meet performance and mass constraints. As part of the continuing analysis, the team is re-assessing initialization methods, considering gyrocompassing analysis as well as external measurements or transfers from the lander systems. In order to understand this impact, a variety of IMU platforms were assessed to provide into their insertion performance for the notional solid vehicle. For this analysis, all baseline dispersions were enabled, and the vehicles were all initialized with a 0.2 degree one-sigma total attitude error. Figures 2629 show the final insertion conditions for 4 IMU platforms: STIM300 (tactical MEMS), LN200S (low-grade navigation), HG5700 (low-grade navigation), and HQ (a MIMU navigation grade unit). In each plot axis, the major gridlines are set at $50 \mathrm{~km}$ intervals.

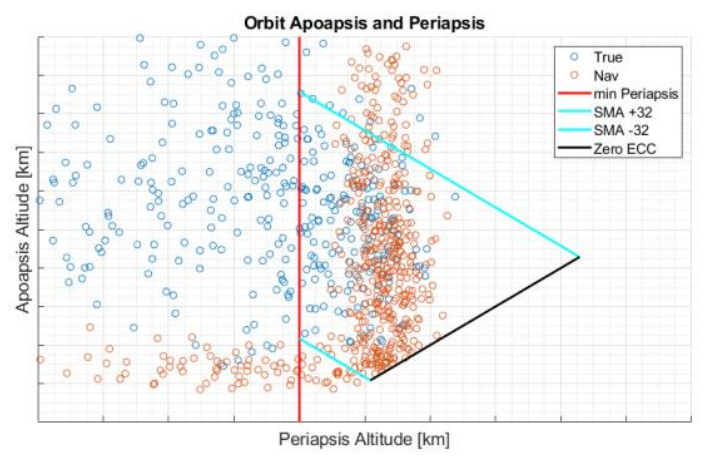

Figure 26: STIM300 w/ .1 Degree Initial Attitude Error

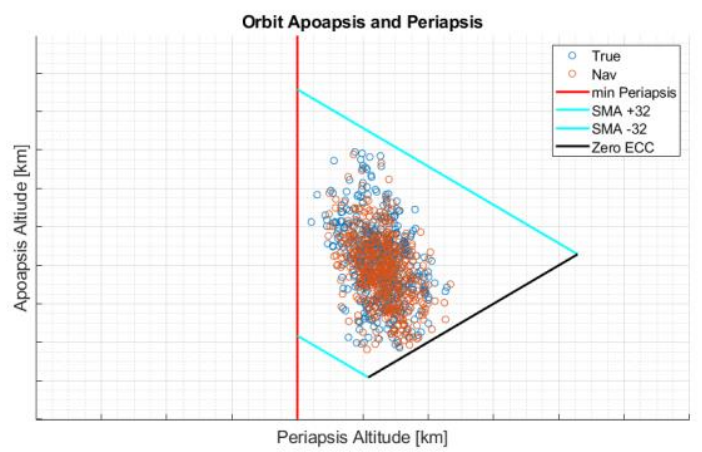

Figure 27: LN200 with .1 Degree Initial Attitude Error

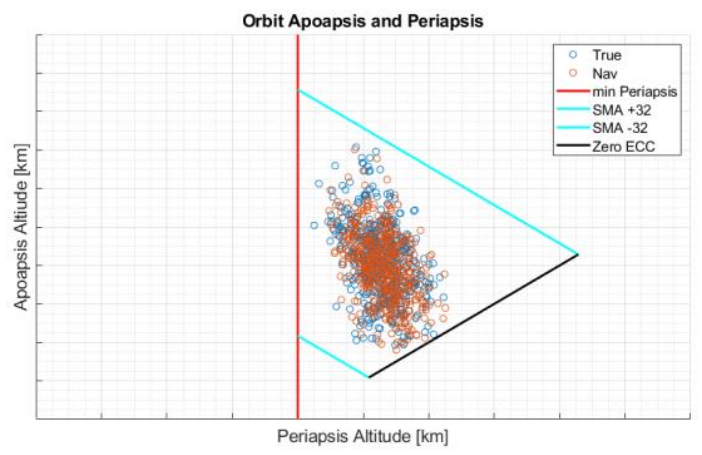

Figure 28: HG5700 with .1 Degree Initial Attitude Error

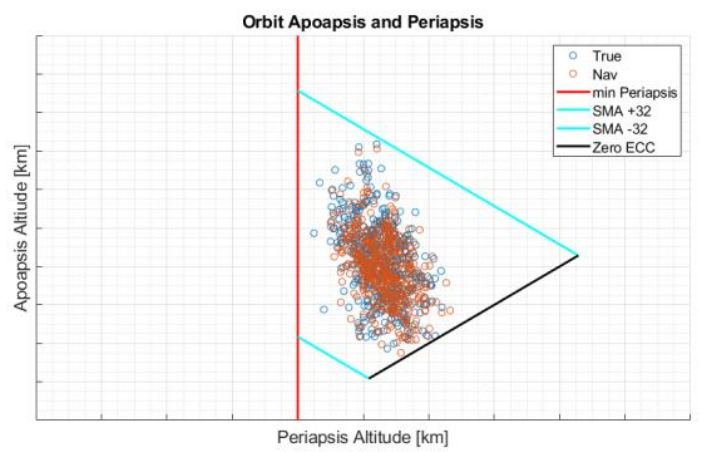

Figure 29: HQ IMU with .1 Degree Initial Attitude Error 
The primary takeaway from the results is the similarity between Figures 27, 28, and 29. As the IMU improves, the insertion capability essentially bottoms out. This does not mean that a high grade navigation unit is not needed. Conversely, it reflects the sensitivities shown previously: initial attitude errors and other vehicle dispersions have a larger effect on insertion capability than navigation errors accumulated over ascent. It also reflects the need to be able to adequately maintain an onboard inertial attitude solution to a certain capability over flight to maintain pointing.

The primary driver for a higher grade navigation system is the ability to grycompass the IMU prior to launch in obtaining the initial attitude. In this scenario, accelerometer bias, gyroscope bias, and gyroscope angular random walk are the primary contributors to the ability for the IMU to discern its attitude. In this case, the initial attitudes uncertainty would vary from that used in this analysis, and more differentiation would be identified between the results. As the vehicle design continues to mature, the initialization algorithms will be refined, implemented, and ground tested to verify insertion performance.

\section{Conclusions}

The inherent differences in functionality between the solid motor vehicle and the hybrid motor vehicle mandate independent guidance algorithms. Figures 1-3 demonstrate the need to address additional energy present in the solid motor vehicle and suggest that employing SXS in a generalized energy management architecture can be an effective way to reduce orbit insertion error caused by dispersions in environment or performance experienced during the first burn.

The Monte Carlo results presented in Figures 19-23 suggest that employing the current GNC architecture, including energy management, provides a design that demonstrates feasibility in responding to dispersions and approaches target orbital accuracy goals. Additionally, the sensitivity analysis performed (both 1-D trades and Monte Carlo) provide a high level of insight into key system sensitivities and help to identify where reducing vehicle dispersions can help to improve insertion accuracy.

\section{FUTURE WORK}

The results of the integrated vehicle analysis identify the clear sensitivity to initial errors, but also the strong sensitivity to mass and thrust uncertainties. While thrust uncertainties are inherent in solid motor design, the future work of the vehicle is focusing on mitigation strategies for further refinement of this vehicle's capability. One approach under development is the implementation of an RCS-based approach to final orbit correction. This helps to reduce the sensitivity to second stage thrust dispersions. Initial analysis shows the DV requirements to be within the capability of the system, but design trades are ongoing for additional thruster integration and placement. Another area of active refinement is the maturation of the ground alignment algorithms. The team is implementing gyrocompassing algorithms to provide a higher level into expected performance above first order estimates based on sensor bias and noise terms. Similarly, the team will be investigating the actual flight platforms, for their operation in the intended environment and performance inhouse calibration and operational activities to validate alignment capability.

\section{ACKNOWLEDGEMENTS}

The team would like to acknowledge the Mars Sample Return Project staff at Marshall Space Flight Center in supporting this analysis and publication. Additionally, the authors must provide recognition to the engineers and scientists at the Jet Propulsion Laboratory, who have spearheaded this effort and lead this program. This research builds on their excellent design and engineering. Additional thanks are directed towards our Engineering department, division, and branch staff, particularly Heather Koehler, for her support of this effort. Also, the team would like to thank Robin Pinson and Naeem Ahmad for their guidance in guidance algorithm development. 


\section{REFERENCES}

[1] Anzalone, Erickson, Montalvo, "Guidance and Navigation Design for a Martian Sample Return Ascent Vehicle," presented at AAS Guidance, Navigation and Control Conference; Breckenridge, CO, United States, February 01 - 06, 2019, AAS 19-034, M19-7315. Available on ntrs.nasa.gov, Document ID: 20190020084

[2] Miller, Steve, and Jeff Wendlandt. "Real-time simulation of physical systems using simscape." MATLAB News and Notes, 2010, pp. 1-13.

[3] Mars-GRAM 2010, "Mars-Global Reference Atmospheric Model," https://software.nasa.gov, MFS-33158-1, msfcsra-team@mail.nasa.gov

[4] POST, "Program to Optimize Simulated Trajectories II (POST2)", https://post2.larc.nasa.gov

[5] Everett, Jason, "A Generalized Guidance Approach to InSpace Solid-Propellant Vehicle Maneuvers,"

[6] Vallado, David,"Orbital Maneuvering," in Fundamentals of Astrodynamics and Applications, $4^{\text {th }}$ ed. Hawthorne, California, United States: Microcosm Press, 2013, ch. 6, sec.6.3-6.4, pp. 320-337

[7] Battin, Richard H., "An Introduction to the Mathematics and Methods of Astrodynamics", AIAA, Reston, VA, 1999, Introduction, Chapter 11.

[8] McHenry R. L., Brand T. J., Long A. D., Cockrell B. F., Thibodeau III J. R., "Space Shuttle Ascent Guidance, Navigation, and Control," The Journal of the Astronautical Sciences, Volume XXVII, No. 1, January-March, 1979, pages $1-38$

\section{BIOGRAPHY}

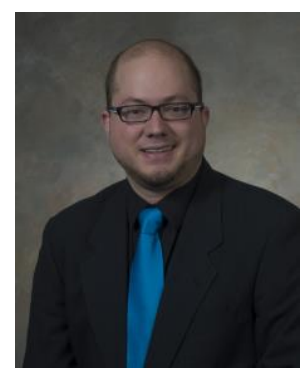

Evan Anzalone received a B.S. in Physics from Louisiana State University in 2006. He completed his Master of Science Degree at Georgia Institute of Technology in 2008, and PhD in Aerospace Engineering in 2013, with his thesis, "Agent and model-based simulation framework for deep space navigation analysis and design." He has been with NASA/MSFC for 9 years. He currently serves as a Navigation SME for the EV42 Guidance, Navigation, and Mission Analysis branch. He co-leads the SLS Navigation Team and supports system architecture, hardware review and specifications, performance analysis, and fault detection and isolation analysis. He is also the inventor and developer of the Multi-spacecraft Autonomous Positioning System, and PI of the Lunar Node -1 payload, which will demonstrate lunar navigation beacon hardware in 2021. In addition to SLS, Evan is also the Navigation lead for the Martian Sample

Return Ascent vehicle work being done at NASA/MSFC.
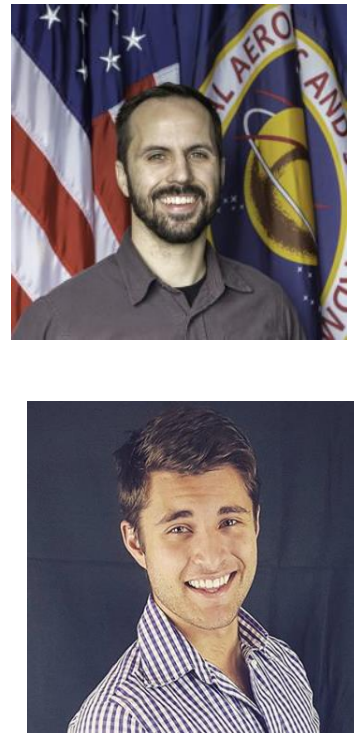

Dane Erickson received a B.S. in Mechanical Engineering from Oregon State University in 2017. He currently supports $3 D O F$, 6DOF, mission analysis, and vehicle design studies in the Guidance, Navigation, and Mission Analysis branch of MSFC.

Jason Everett received a B.S. in Aerospace Engineering from The Pennsylvania State University in May 2018. Since his start in civil service at NASA's Marshall Space Flight Center, he has been working as a full time GNC engineer for several of NASA's projects including the Mars Ascent Vehicle, the Space Launch System, two solar sail missions, and two lunar lander designs. In September 2018, he received a Center Director's Special Service Award by supporting standalone GNC implementation for SLS critical liftoff and tower clearance assessments. He currently supports flight readiness analysis for the SLS Artemis I mission.

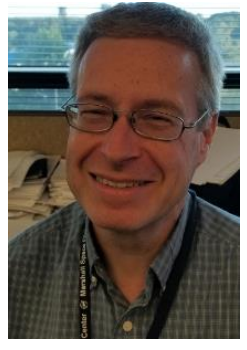

Joey Powers received a Bachelor of Aerospace Engineering (BAE) degree in 1990 and a Master of Aerospace Engineering (MAE) degree in 1993 from Auburn University. In 1985, he began working as a co-op student at the NASA Marshall Space Flight Center in Huntsville, AL. Upon receiving his $B A E$, Mr. Powers began working full time at MSFC in 1990. Currently, he works in the Control Systems Design and Analysis Branch/EV41 at MSFC. In his 32 years of service time working at MSFC, Mr. Powers has been performing flight vehicle controls design and analysis work. He has performed controls system analysis/evaluation and design work on several projects including Small Expendable Deployable Satellite (SEDS), Bantam, X37, Ares I, Ares V, Space Launch System (SLS) and various launch vehicle concepts. Mr. Powers also has supported the development of various time and frequency domain software tools used in the design and analyses of flight vehicles such as the six-degree-of-freedom simulation Marshall Aerospace Vehicle Representative In $C$ (MAVERIC). Mr. Powers is currently the MSR MAV GN\&C lead at MSFC. 